\title{
ORIGINAL ARTICLE \\ Could motor imagery be effective in upper limb rehabilitation of individuals with spinal cord injury? A case study
}

\author{
M Grangeon ${ }^{1,2,3}$, P Revol ${ }^{4}$, A Guillot ${ }^{1,5}$, G Rode ${ }^{4}$ and C Collet ${ }^{1}$
}

Study design: A case study.

Objective: The aim was to investigate whether motor imagery $(\mathrm{MI})$ could be successfully incorporated into conventional therapy among individuals with spinal cord injury (SCI) to improve upper limb (UL) function.

Setting: The Physical Medicine and Rehabilitation Unit at the Henry Gabrielle Hospital in Lyon, France.

Methods: The participant was an individual with a complete C6 SCl. MI content was focused on functional UL movements, to improve hand transport to reach out and grasp with tenodesis. The participant was tested before and after $15 \mathrm{MI}$ training sessions ( 45 min each, three times a week during 5 consecutive weeks). MI ability and program compliance were used as indicators of feasibility. The Minnesota and Box and Blocks tests, as well as movement time and hand trajectory during targeted movements were the dependent variables, evaluating motor performance before and after MI training.

Results: The participant's ability to generate MI was checked and compliance with the rehabilitation program was confirmed. The time needed to complete the Minnesota test decreased by 1 min $25 \mathrm{~s}$. The Box and Blocks score was improved by three units after MI program. Decreased movement time and enhanced hand trajectory smoothness were still observed 3 months later, despite a slight decrease in performance.

Conclusions: This study supports the feasibility for introducing MI in conventional therapy. Further studies should confirm the potential role of $\mathrm{MI}$ in motor recovery with a larger sample.

Spinal Cord (2012) 50, 766-771; doi:10.1038/sc.2012.41; published online 17 April 2012

Keywords: motor imagery; spinal cord injury; tenodesis; reaching; hand transport; rehabilitation

\section{INTRODUCTION}

Individuals with cervical spinal cord injury (SCI) experience varying degrees of sensorimotor impairments of the upper limbs (ULs), lower limbs and trunk. The loss of UL function, especially grip, may have deleterious consequences in everyday life. Improving hand function is thus one of the main objectives during the rehabilitation process. Traditional therapy frequently consists of physical repetition, ${ }^{1}$ orthotic use, $^{2}$ or UL surgery. ${ }^{3}$ Integrating mental rehearsal based on motor imagery (MI) into conventional therapy was aimed at improving rehabilitation efficiency. $\mathrm{MI}$ is the mental representation of an action without any overt movement. One of the most remarkable features of $\mathrm{MI}$ is that it shares common neuronal networks with actual execution. In other words, MI has the ability to activate the neural networks controlling the planning and programming of movement, thus maintaining active the motor programs stored in the procedural memory. In the clinical context, there is now compelling evidence that MI combined with physical practice is more effective than physical or mental practice alone. In fact, combining MI with actual repetitions $s^{4,5}$ may help to create accurate and vivid mental images due to the sensory feedback originating from movement, even passively guided (mainly from the visual system in case of de-afferentation after SCI).

MI should be effective owing to the activation of neuronal networks that are similar to those activated during actual execution. ${ }^{6}$ Caution must however be exercised before drawing final conclusions, as a recent review ${ }^{7}$ provided limited evidence about the effectiveness of MI in UL function training following stroke and suggested the need to further investigate the potential benefits of MI in clinical rehabilitation. Besides numerous post-stroke studies, the integration of MI in SCI rehabilitation received limited attention. Numerous neuroimaging studies demonstrated that mental representation is retained in individuals with $\mathrm{SCI}^{8}$ Cramer et al..$^{9}$ identified the activation of similar areas that are involved in the learning process, both in healthy controls and individuals with SCI, during MI training of lower limb movements. They also showed that MI training similarly improved behavioral outcomes and tongue movement speed in both groups. These findings suggest that the neural networks controlling movement might be centrally activated above and below the lesion level, even in the absence of peripheral feedback.

${ }^{1}$ Université de Lyon, Université Claude Bernard Lyon1-CRIS EA 647-Laboratoire de la Performance Motrice, Mentale et du Matériel (P3M) Villeurbanne, France; ${ }^{2}$ School of Rehabilitation, Université de Montréal, Montreal, Quebec, Canada; ${ }^{3}$ Centre for Interdisciplinary Research in Rehabilitation of Greater Montreal - Rehabilitation Institute Gingras-Lindsay-de-Montréal, Laboratory of Pathokinesiology, Montreal, Quebec, Canada; ${ }^{4}$ Université de Lyon, Université Lyon 1, INSERM-UMRS 534, Bron, \& Hospices Civils de Lyon, Plate-forme « Mouvement et Handicap » Hôpital Henry Gabrielle, Lyon, France and ${ }^{5}$ nnstitut Universitaire de France, Paris, France

Correspondence: Professor Dr C Collet, Université de Lyon, Université Claude Bernard Lyon1 - CRIS EA 647 - Performance Motrice, Mentale et du Matériel (P3M) 27-29 Boulevard du 11 Novembre 1918, 69622 Villeurbanne Cedex, France.

E-mail: christian.collet@univ-lyon1.fr.

Received 3 November 2011; revised 15 March 2012; accepted 16 March 2012; published online 17 April 2012 
Despite these encouraging results, the integration of MI in SCI rehabilitation programs for improving functional recovery has not yet been experimentally addressed. The aim of this case study was to assess the feasibility of a rehabilitation program combining MI practice and conventional therapy for improving UL function in individuals with SCI, using (1) rehabilitation program compliance and (2) quantitative assessments of UL performance. As brain structures were spared by the injury, we postulated that the participant would be able to generate vivid and accurate images despite the impairment due to SCI, and complete the whole MI program. We further expected that kinematic analysis and functional tests would be potential indicators of UL function improvement in future studies.

\section{PATIENTS AND METHODS}

\section{Participant}

The participant voluntarily took part in this study and gave his informed consent after the medical team and the local human research ethics committee approved the experiment. Then, we verified that he had enough abilities in forming mental images for the protocol to be completed. He was 23 years old, right handed ${ }^{10}$ and had sustained a traumatic SCI due to a C6 burst fracture 8 months earlier. He underwent surgery with reduction and $\mathrm{C} 5-\mathrm{C} 7$ osteosynthesis on the day of accident. He was not comatose and did not sustain any brain injury, although the scan did reveal hemorrhagic damage at the C6 level. The neurological check-up was computed by a physiotherapist and showed infra-lesional motor and sensory deficits at the C6 level. Motor, sensory and light touch (pinprick sensation) scores were 22, 24 and 28, respectively, using the ASIA (American Spinal Injury Association) Impairment scale (AIS). The SCI was classified as AIS A owing to the absence of motor and sensory function in sacral segments S4-S5. Manual testing of the right UL showed normal strength in shoulder muscles and elbow flexors. Triceps brachii function was altered and rated as 2 on a 5 -level scale. There was general paralysis of the flexor carpi radialis and hand muscles. The participant displayed moderate spasticity without elbow and wrist movement restriction.

\section{Interventions}

When the experiment started, the patient had already received conventional physiotherapy and demonstrated stable UL functional scores. Thus, performance did not change from the end of therapy until the present baseline measurements 10 days after. Each training session was a combination of conventional therapy and MI repetitions, conducted by the same physiotherapist under similar conditions. The experimental design lasted 5 consecutive weeks. The MI/physical practice ratio increased from 5:1 to 10:1, as the participant was able to withstand increased mental load due to progress in training. The total number of repetitions also increased over the 5-week period (Table 1). During the first week, the participant completed functional and mental ability tests and kinematic evaluations under the supervision of an external examiner. We scheduled a specific MI training program between weeks 2 and 5, to improve hand movement towards target to reach out and grasp various objects using tenodesis. Then, a post-training test was performed and two retention tests were planned 1 and 3 months later. All interventions were conducted at the Rehabilitation Hospital Unit.

The 5-week training program consisted of three weekly sessions of $45 \mathrm{~min}$ each. Physical training was only used to facilitate the imagined movement by providing visual and kinesthetic feedback. Consequently, the expected improvement in motor skills (if observed) would mainly result from the mental rehearsal and not from actual trials. Both visual and kinesthetic MI were believed to provide useful feedback to the participant in controlling movement execution. Visual MI involved self-visualization of action stages based on visual cues; kinesthetic MI involved imagining the feeling of performing the movement. The participant was instructed to close his eyes during MI to better focus on movement accuracy. The training sequences consisted of reaching and grasping tasks based on daily living activities. The participant was asked to self-evaluate his ability every four MI trials to create accurate mental images on a 4-point Lickert scale (where 1 represented very poor imagery and 4 represented vivid imagery) and to ensure that instructions were adequately followed. At the end of each session, we interviewed the participant about his own perception of the MI program to ensure that it was well adapted to his actual abilities, and took his improvement into account. When applicable, it was adjusted at the next session. Program compliance was recorded in a logbook.

Table 1 Summary of the training program and description of actual tasks

\begin{tabular}{|c|c|c|c|c|c|c|c|c|c|c|c|c|c|c|c|c|}
\hline & Session & 1 & 2 & 3 & 4 & 5 & 6 & 7 & 8 & 9 & 10 & 11 & 12 & 13 & 14 & 15 \\
\hline \multirow[t]{3}{*}{ Initial training } & MI trials & 15 & 18 & 18 & 24 & 24 & 24 & 27 & 27 & 27 & 30 & 30 & 30 & 30 & 32 & 35 \\
\hline & PP trials & 3 & 3 & 3 & 3 & 3 & 3 & 3 & 3 & 3 & 3 & 3 & 3 & 3 & 4 & 5 \\
\hline & Ratio MI/PP & $5: 1$ & $6: 1$ & $6: 1$ & $8: 1$ & $8: 1$ & $8: 1$ & $9: 1$ & $9: 1$ & $9: 1$ & $10: 1$ & 10:1 & 10:1 & $10: 1$ & $8: 1$ & $7: 1$ \\
\hline \multirow[t]{3}{*}{ Effective training } & MI trials & 15 & 18 & 18 & 24 & 24 & 24 & 27 & 27 & 7 & 30 & 14 & 30 & 30 & 32 & 13 \\
\hline & PP trials & 3 & 3 & 3 & 3 & 3 & 3 & 3 & 3 & 1 & 3 & 2 & 3 & 3 & 4 & 2 \\
\hline & MI/PP Ratio & $5: 1$ & $6: 1$ & $6: 1$ & $8: 1$ & $8: 1$ & $8: 1$ & $9: 1$ & $9: 1$ & $7: 1$ & $10: 1$ & $10: 1$ & 10:1 & $10: 1$ & $8: 1$ & $6: 1$ \\
\hline
\end{tabular}

Description of

actual tasks

Reach out the top of each object at various heights in IL and $\mathrm{CL}$ space
Grasp the rings varying in weight in front of you and transfer them to the horizontal $\mathrm{IL}$ and $\mathrm{CL}$ target
Grasp the objects placed in front of you (IL and CL space) and transfer them to each target varying in height and direction ( $0^{\circ}$ to $180^{\circ}$ horizontal plane).
Reach out to grasp the object located in the CL space and transfer it to the IL target. Then transfer the objects located in the IL space to the $\mathrm{CL}$ target.

The shape of the objects and the height of the target change from one session to another.
Complexity of the above tasks

Reach out the top of each object
by transferring your hand just
above the horizontal line
located at a height
of $20-25 \mathrm{~cm}$

Grasp the rings in front of you and transfer them to an $\mathrm{IL}$ and $\mathrm{CL}$ vertical target with respect to hand transport just above the horizontal line located at a height of $20-25 \mathrm{~cm}$.
Try to decrease the duration of movement across trials in the same session. The shape and weight of the object differ from one session to another. 


\section{Measures}

MI ability. MI is a complex and covert process, and due to its concealed nature, MI vividness and accuracy remain rather difficult to evaluate. However, we used a set of tests integrated into an original index to evaluate the participant's ability to generate imagined movements ${ }^{11}$ : (i) KVIQ (Kinesthetic and Visual Imagery Questionnaire) ${ }^{12}$ to evaluate MI vividness; (ii) mental chronometry test to evaluate the preservation of temporal organization of the imagined movement, ${ }^{11,13}$ and (iii) electrodermal response (EDR) analysis to evaluate the mental effort during MI. ${ }^{11,14}$

The KVIQ allowed for the assessment of 10 movements of different body segments. A 5-point rating scale enabled the calculation of the KVIQ score $(1=$ low level of imagery and $5=$ high level of imagery). The participant was asked to physically perform each movement from a sitting position and then imagine the same action by using visual and kinesthetic MI. As actual kinesthetic feedback was probably altered, the participant may have recalled information from long-term memory cues, using a top-down process. In this case, it was supposed that he created the motor image from the expected kinesthetic feedback stored in his long-term memory. When he could not perform the movement, he observed the movement being performed by the examiner before engaging in MI.

Mental chronometry evaluated the ability to achieve temporal congruence between the imagined and actual movement by comparing the duration of the physical movement to that of MI. EDR, an index of the sympathetic nervous system, was used as the third indicator of MI ability simultaneously to mental chronometry. Previous results reported strong relationships between EDR and mental processes. ${ }^{14} \mathrm{MI}$ was considered accurate when EDR matched that of actual execution (Figure 1). Mental chronometry and EDR were analyzed during the movement of reaching out to grasp a glass (see detailed information in the kinematic measures section). Combining these three indices provided a thorough procedure for assessing MI ability, ${ }^{11}$ as this should be considered before enrolling a participant in a MI program.?

Functional movement assessments. Manual dexterity was tested using the Box and Blocks ${ }^{15}$ and Minnesota tests, ${ }^{16}$ which are well-known indicators of UL function performance. To prevent from habituation and learning, the participant performed only one trial during each evaluation session.

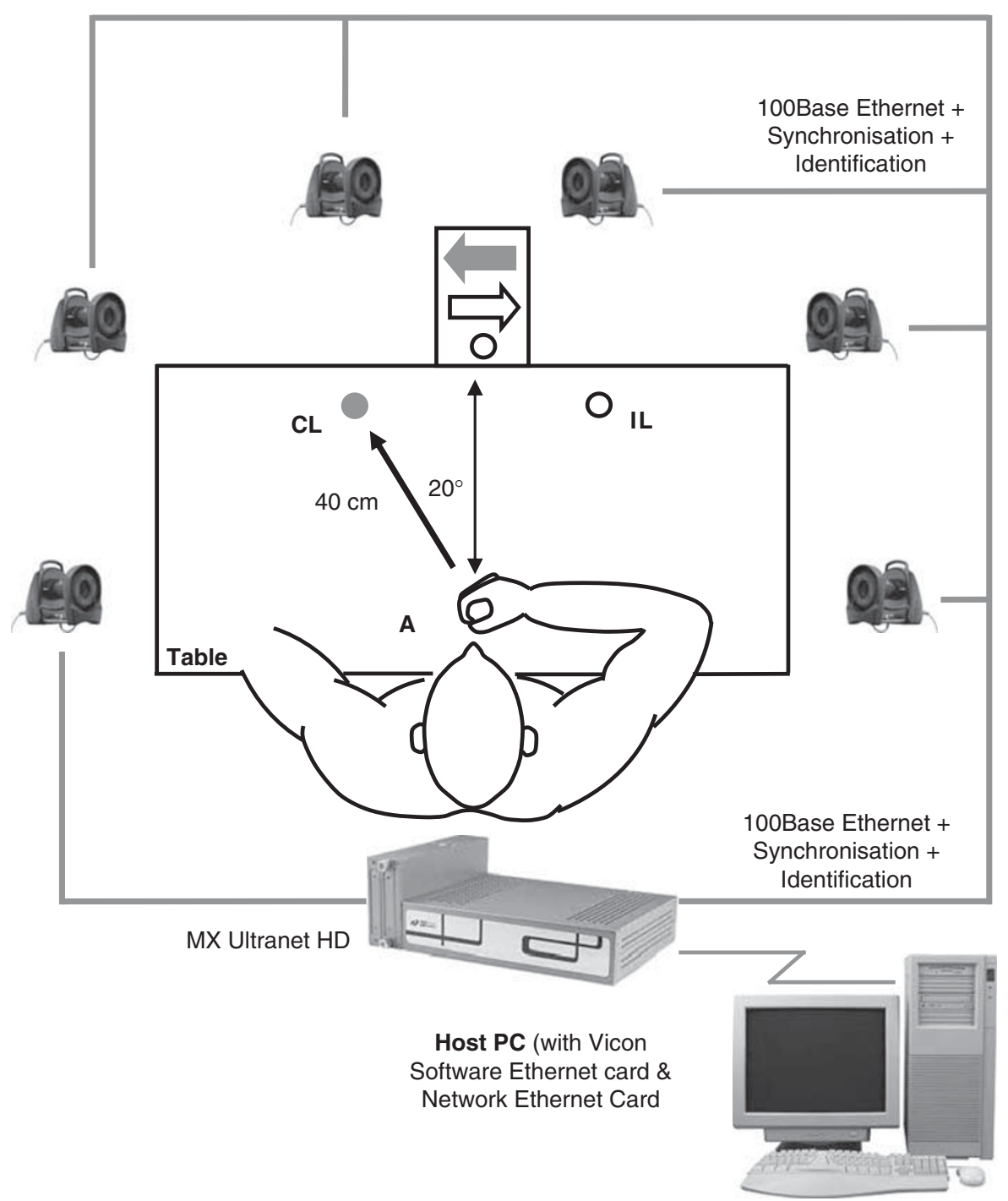

Figure 1 Experimental design. The height of the table was set up to fit the patient's morphological features. The point A indicates the starting point of the hand, whereas the arrow shows the glass to reach and grasp (gray circles). The Vicon MX system recorded kinematics of hand trajectory using retro-reflective markers attached to the wrist, the forefinger and the thumb of the dominant hand. The infra-red light cameras gave a clear grayscale view from the markers used to compute the co-ordinates of each marker. The system integrated co-ordinates from all cameras and tracked the markers automatically to compute accurate 3D trajectories. IL: ipsilateral space; CL: controlateral space. 


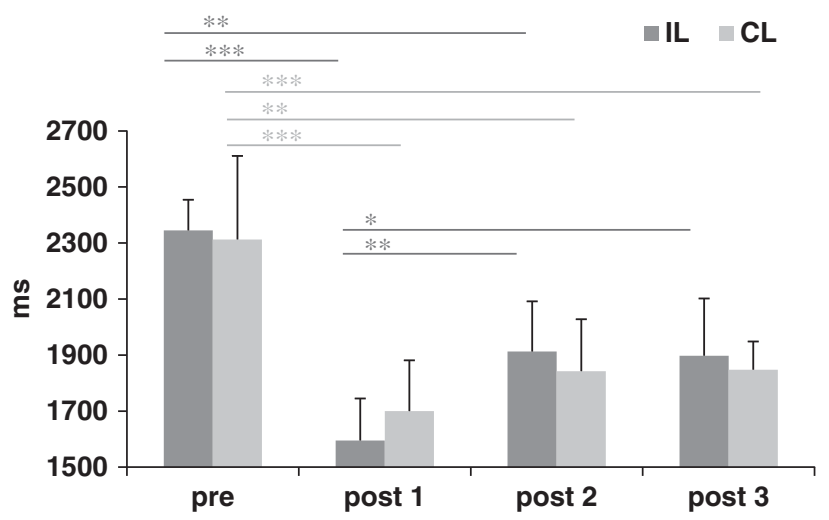

Figure 2 Variations of movement time between the sessions testing the action of grasping the glass in the IL space and in the CL space. Post 1 , 2 and 3: post-test (post 1) and retention tests after 1 and 3 months (post 2 and 3 , respectively). Movement time decreased and then slightly increased during the retention period but remained lower by comparison with the pre-test, especially in the IL movement. ${ }^{* *} P<0.001 ;{ }^{*} P=0.01 ;{ }^{*} P=0.05$.

The first test required the participant to grasp and transfer individual blocks within a partitioned box using the dominant and then the non-dominant hand for a 60 -s time period. The number of blocks successfully transferred was the dependent variable. The Minnesota test required the participant to place 60 disks into holes in a board while using a wide range of unilateral and bilateral shoulder movements. ${ }^{14}$ The time taken to complete the test was the dependent variable.

Kinematic measures. The participant sat in a wheelchair and was kept motionless by a strap across his chest. When the onset signal indicated the target to be reached, the participant was asked to reach out and grasp the glass to simulate drinking before returning the glass back to the starting position. The glass was placed in the participant's ipsilateral (IL) or controlateral (CL) haptic space. The participant had thus to reach out the glass with his dominant hand in two directions, that is, left and right, which respectively corresponded to the controlateral and ipsilateral spaces. In all, 20 trials were randomly performed in each direction and were video-recorded using the 3D Vicon MX system (Oxford, UK) placed above the participant to record data from the horizontal plane (Figure 2). Kinematic measures started at the beginning of each movement and ended as soon as the patient grasped the glass. Movement duration and smoothness of the wrist trajectory were considered the dependent variables, that is, indicators of the quality of the movement to reach out to grasp using tenodesis. ${ }^{17}$ Smoothness was defined by the variability in the horizontal plane and the total distance covered by the wrist.

Functional scores and trajectory smoothness were analyzed with descriptive statistics. The functional scores and kinematic measures were considered performance indices from the pre-test to the post-test.

\section{RESULTS}

The visual KVIQ score was 70 out of 85 , whereas the kinesthetic score was 57, suggesting greater visual imagery than kinesthetic imagery ability. We found a significant correlation between actual and imagined movement times $\left(r^{2}(10)=0.91 ; P=0.003\right)$ as well as between EDR duration under the same conditions $\left(r^{2}(10)=0.72\right.$; $P=0.02$ ), demonstrating that the participant was able to achieve temporal congruence and accurate MI. The logbook data indicated that the participant performed 342 MI trials out of the 400 previously planned. Accordingly, because of shoulder pain during two sessions, the participant did not perform all MI trials (194 and 148 out of 200 trials, respectively, under the CL and IL conditions). Self-report ratings and interviews further suggested that he did not encounter any difficulty when imagining the movements (3.44 and 3 in visual and
Table 2 Functional improvement after motor imagery training

\begin{tabular}{llcccc}
\hline Test & Side & Pre-test & Post 1 & Post 2 & Post 3 \\
\hline Minnesota (minute) & Trained side & 3.33 & 2.07 & 2.04 & 2.02 \\
& Untrained side & 2.41 & 2.36 & 2.26 & 2.30 \\
& Bilateral movement & 6 & $7.3^{3}$ & 5.51 & 5.32 \\
Box and blocks (number) & Trained side & 25 & 28 & 28 & 29 \\
& Untrained side & 26 & 22 & 26 & 24
\end{tabular}

Shoulder pain altered performance.

Scores of the dominant hand improved in both the Minnesota and the Box and Blocks tests. Post 1, 2 and 3: post-test (post 1) and retention tests after 1 and 3 months (post 2 and 3, respectively).

kinesthetic MI, respectively), and the difficulty of the MI program was deemed to be adjusted to his physical and cognitive abilities.

The time needed to complete the Minnesota test decreased by $1 \mathrm{~min} 25 \mathrm{~s}$. The Box and Blocks score was improved by three units (Table 2). Interestingly, the participant's performance remained stable 3 months after the training program. After MI training, the score in the Box and Blocks test using the trained UL outperformed that of the untrained UL, that is, +3 and -4 , respectively. However, we observed a great variability of performance in the untrained UL.

Movement time and trajectory smoothness improved following MI training (Figures 2 and 3). IL movement was $1595 \mathrm{~ms}$ during the post-test, that is, $32 \%$ faster than the pre-test. CL movement time was $1700 \mathrm{~ms}$ in the post-test, that is, $26.5 \%$ faster than the pre-test.

The variability of wrist trajectory decreased in both IL $(-8.52 \mathrm{~mm})$ and CL movements $(-8.32 \mathrm{~mm})$, that is, an improvement of $66 \%$ and $50 \%$, respectively. Therefore, the distance covered by the wrist also decreased by $9 \%$ and $3 \%$ in terms of IL $(-30 \mathrm{~mm})$ and CL $(-10 \mathrm{~mm})$ movements, respectively. Kinematic measures again remained stable after 3 months. However, movement time and trajectory smoothness slightly increased during the retention phase, particularly in IL movement, but performance remained substantially better than before MI training.

\section{DISCUSSION}

Although little data support the integration of MI in rehabilitation programs for individuals with SCI, present results show a feasible way to consider the therapeutic benefits of MI. The kinematic assessments support the potential benefit of MI training for UL function improvement, although future studies with larger sample size are required. Learning tenodesis required the participant to change motor programming of reaching out. The new muscle coordination involved (i) a complex UL coordination to extend the elbow to compensate for the weakened triceps brachii function, and (ii) hand transport control during the reaching phase to place the hand above the glass allowing for tenodesis grasp. ${ }^{17}$ Smoothness trajectory and movement duration improvement after MI training confirmed the spatial reorganization of hand transport during the reaching phase and suggested better control of arm direction. According to Laffont et al., ${ }^{18}$ reaching out to grasp requires lateral rotation and supination of the scapula in individuals with SCI at the C6 level, and weaker involvement of the glenohumeral joint in the external workspace. Thus, the ballistic phase remains possible using gravity and the interaction of dynamic forces between the shoulder and the elbow.

The slight increase in movement time in the IL space may be explained by the characteristics of the movement. Indeed, Tseng and Scholtz ${ }^{19}$ demonstrated that pointing towards a target resulted in shorter movement time and more accurate pointing when performed controlaterally compared with an IL movement. Additionally, the 


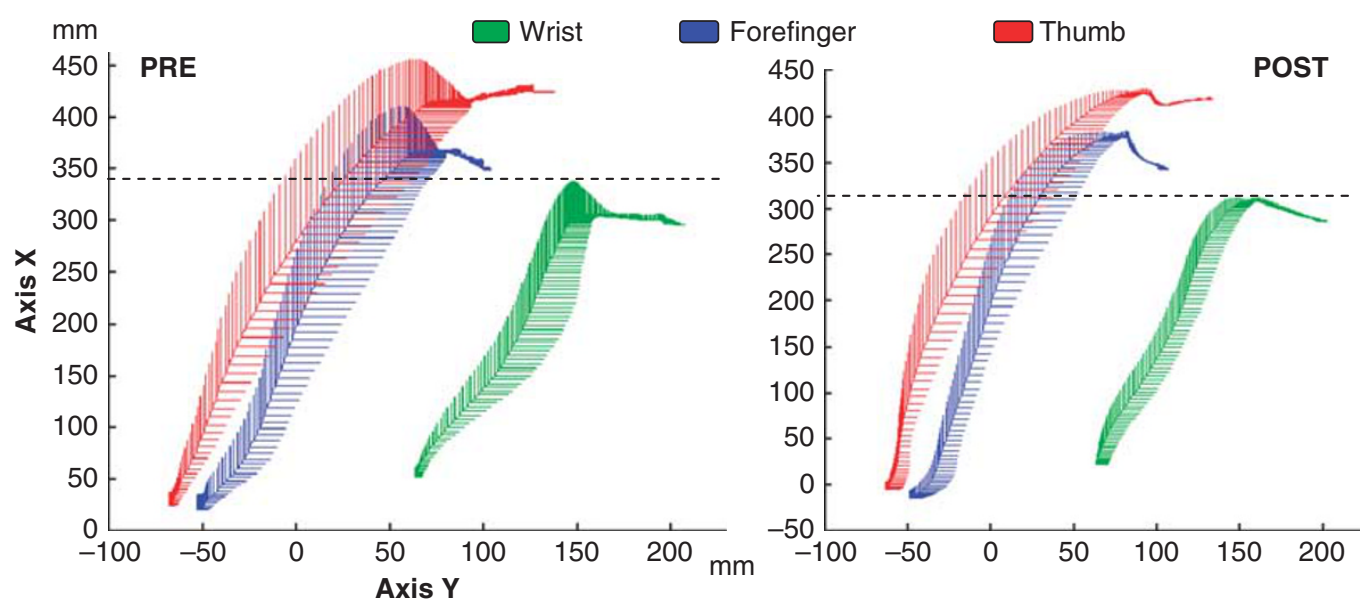

Reaching out in the ipsilateral space
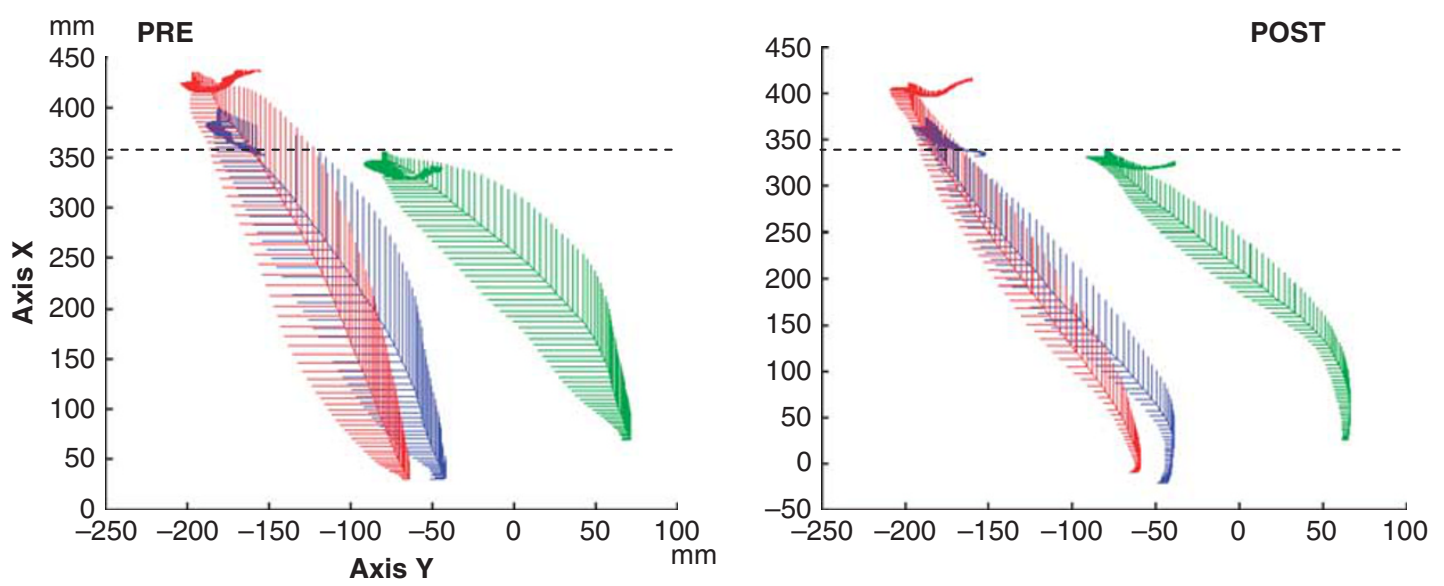

Reaching out in the controlateral space

Figure 3 Hand trajectory $(\mathrm{mm})$ in the horizontal plane before and after MI training, during the IL (top) and CL movement (bottom). The hand trajectory was analyzed through the wrist marker, as the patient could not move the fingers owing to paralysis. The variability decreased during the post-test particularly at the end of the movement, the part of reaching, which is controlled by sensory feedback. The distance covered by the hand in the sagittal plane was shorter after MI training (dotted line).

amount of practice was not equal between IL and CL movements owing to the participant's shoulder pain. The effect of MI might be explained by the feedback provided during actual practice, which may help the retention of motor programming by reinforcing recognition and recall from procedural memory. ${ }^{20}$ Strengthening a new motor skill requires a minimum of rehearsal. The weaker amount of trials in the IL space (owing to chronic pain), could probably explain why performance was lower by comparison with the CL space. Although the inclusion of little physical practice has been validated in earlier studies, ${ }^{4,5}$ the proportion of MI vs physical practice still needs to be better determined. Malouin et al. ${ }^{21}$ and Jackson et al., ${ }^{4}$ respectively, included 1100 and $1500 \mathrm{MI}$ trials in their rehabilitation program vs 194 and 148 for the CL and IL movements in the present study. Further testing is therefore needed to determine the extent to which a tailored MI program may better consolidate the performance level and indicate the optimal ratio between MI and actual practice.

Additionally, the score obtained with the Box and Blocks and Minnesota tests during post-training confirmed the improvement of UL function performance. However, the great variability in the untrained limb remains questionable. The transfer of motor learning between ULs is usually observed across the two ULs in reaching and catching tasks. ${ }^{22,23}$ However, the participant in our experiment did not benefit from this transfer effect. The period of observation may have been too short to observe functional difference, which could be recorded with kinematics tools. Another working hypothesis awaiting further investigation could be the dissymmetry of the SCI, causing functional motor deficits, which are more difficult to treat. The use of functional assessments specifically designed to evaluate individuals with SCI should also be considered in future studies (for example, Graded Redefined Assessment of Strength, Sensibility and Prehension - GRASSP $^{24}$ ). The transport phase and the grasping phase with tenodesis are the two main components enabling catching within the haptic space. Learning tenodesis requires changing the transport phase as the hand should be placed above the object to grasp, due to wrist extension. Thus, the first step in the rehabilitation of grasping after SCI is to change the transport phase of the arm toward the target with the aim to favor grasping with tenodesis, which requires the prior extension of the wrist, needed for closing the fingers on the palm.

Lastly, compliance with the MI program was maintained as the participant completed almost all sessions and his MI ability was confirmed despite sensorimotor impairment due to SCI. However, he 
did not complete three training sessions as pain increased during MI trials. MI, which activates similar cortical pathways to actual execution, might involve brain circuits mediating pain processing, independently from peripheral inputs. This analysis underscores the need for close clinical supervision when undertaking a MI program and for treatment based on participants' characteristics. Additionally, interviews with the participant confirm that the MI program was not found to be boring, due to the various grasping tasks proposed during practice. He perceived the increased difficulty between sessions to be challenging and as a great source of motivation.

To conclude, this study supports the feasibility of introducing MI in conventional therapy to improve UL function after SCI. As expected, the participant was able to form accurate MI and completed almost all sessions. The kinematic and functional assessments confirmed that MI could be associated with conventional therapy for UL improvement in rehabilitation programs.

We should nevertheless underline several limits in this study. Results from clinical trials should be generalized with extreme caution. First, future studies should confirm these results with larger samples. Physiotherapists should also control the ability of the SCI participants to form accurate mental images of movements. This is a crucial point guaranteeing the effectiveness of the MI rehabilitation process. Finally, we have to progress in determining the ratios between mental and physical practices, taking into account the exact nature of the injury, motor and mental skill abilities at any time of the rehabilitation process as well as the adherence and motivation of the individuals for such work.

\section{DATA ARCHIVING}

There were no data to deposit.

\section{CONFLICT OF INTEREST}

The authors declare no conflict of interest.

1 Curtin M. Development of a tetraplegic hand assessment and splinting protocol. Paraplegia 1994; 32: 159-169.

2 Wise MF, Wharton WG, Robinson TD. Long-term use of function hand orthoses by quadriplegics. ASIA Bull 1986; 14: 4-5.
3 Gellman H, Kan D, Waters RL, Nicosa A. Rerouting of the biceps brachii for paralytic supination contracture of the forearm in tetraplegia due to trauma. J Bone Joint Surg Am 1994; 76: 398-402.

4 Jackson PL, Doyon J, Richards CL, Malouin F. The efficacy of combined physical and mental practice in the learning of a foot-sequence task after stroke: a case report. Neurorehabil Neural Repair 2004; 18: 106-111.

5 Malouin F, Richards CL, Durand A, Doyon J. Added value of mental practice combined with a small amount of physical practice on the relearning of rising and sitting poststroke: a pilot study. J Neurol Phys Ther 2009; 33: 195-202.

6 Neuper C, Pfurtscheller G. Electroencephalographic characteristics during motor imagery. In: Guillot A and Collet C (eds) The Neurophysiological Foundations of Mental and Motor Imagery. Oxford University Press Inc., New York, 2010, pp 65-81.

7 Barclay-Goddard RE, Stevenson TJ, Poluha W, Thalman L. Mental practice for treating upper extremity deficits in individuals with hemiparesis after stroke. Cochrane Database Syst Rev 2011, 5: CD 005950.

8 Alkadhi H, Brugger P, Boendermaker SH, Crelier G, Curt A, Hepp-Reymond MC et al. What disconnection tells about motor imagery: evidence from paraplegic patients. Cerebral Cortex 2005; 15: 131-140.

9 Cramer SC, Orr EL, Cohen MJ, Lacourse MG. Effects of motor imagery training after chronic, complete spinal cord injury. Exp Brain Res 2007; 177: 233-242.

10 Oldweld R. The assessment and analysis of handedness: the Edinburg Inventory. Neuropsychologia 1971; 9: 97-113.

11 Collet C, Guillot A, Lebon F, Maclntyre T, Moran A. Measuring motor imagery using psychometric, behavioral, and psychophysiological tools. Exerc Sport Sci Rev 2011; 39: 85-92.

12 Malouin F, Richards CL, Jackson PL, Lafleur MF, Durand A, Doyon J. The Kinesthetic and Visual Imagery Questionnaire $(K V I Q)$ for assessing motor imagery in persons with physical disabilities: a reliability and construct validity study. J Neurol Phys Ther 2007; 31: 20-29.

13 Guillot A, Collet C. Duration of mentally simulated movement: a review. J Motor Behav 2005; 37: 10-20.

14 Guillot A, Haguenauer M, Dittmar A, Collet C. Effect of a fatiguing protocol on motor imagery accuracy. Eur J Appl Physiol 2005; 95: 186-190.

15 Mathiowetz V, Volland G, Kashman N, Weber K. Adult norms for the Box and Block Test of manual dexterity. Am J Occup Ther 1985; 39: 386-391.

16 American-Guidance-Service Minnesota rate of manipulation, examiner's manual. Circle Pines, MN Author, Minnesota, 1957.

17 Hoffmann G, Laffont I, Roby-Brami A. Coordination of reaching movements in patients with a cervical spinal cord injury. Curr Psychol Cogn 2002; 21: 305-340.

18 Laffont I, Briand E, Dizien O, Combeaud M, Bussel B, Revol M et al. Kinematics of prehension and pointing movements in C6 quadriplegic patients. Spinal Cord 2000; 38: 354-362.

19 Tseng YW, Scholz JP. The effect of workspace on the use of motor abundance. Motor Control 2005; 9: 75-100.

20 Taktek K, Zinsser N, St-John B. Visual versus kinesthetic mental imagery: efficacy for the retention and transfer of a closed motor skill in young children. Can J Exp Psychol 2008; 62: 174-187.

21 Malouin F, Belleville S, Richards CL, Desrosiers J, Doyon J. Working memory and mental practice outcomes after stroke. Arch Phys Med Rehabil 2004; 85: 177-183.

22 Malfait N, Shiller DM, Ostry DJ. Transfer of motor learning across arm configurations. J Neurosci 2002; 22: 9656-9660.

23 Morton SM, Lang CE, Bastian AJ. Inter- and intra-limb generalization of adaptation during catching. Exp Brain Res 2001; 141: 438-445.

24 Kalsi-Ryan S, Curt A, Fehlings MG, Verrier MC. Assessment of the hand in tetraplegia using the Graded Redefined Assessment of Strength, Sensibility and Prehension (GRASSP): impairment versus function. Top Spinal Cord Injury Rehabil 2009; 14: 34-46. 